\title{
Stable rank and real rank of compact transformation group $C^{*}$-algebras
}

\author{
by \\ Robert J. Archbold (Aberdeen) and \\ Eberhard Kaniuth (Paderborn)
}

\begin{abstract}
Let $(G, X)$ be a transformation group, where $X$ is a locally compact Hausdorff space and $G$ is a compact group. We investigate the stable rank and the real rank of the transformation group $C^{*}$-algebra $C_{0}(X) \rtimes G$. Explicit formulae are given in the case where $X$ and $G$ are second countable and $X$ is locally of finite $G$-orbit type. As a consequence, we calculate the ranks of the group $C^{*}$-algebra $C^{*}\left(\mathbb{R}^{n} \rtimes G\right)$, where $G$ is a connected closed subgroup of $\mathrm{SO}(n)$ acting on $\mathbb{R}^{n}$ by rotation.
\end{abstract}

Introduction. For a $C^{*}$-algebra $A$, the stable rank $\operatorname{sr}(A)$ was introduced by Rieffel [24] with a view to applications in $K$-theory. In particular, the condition that $\operatorname{sr}(A) \leq 2$ is relevant for cancellation in $K_{0}(A)[2,25]$. The real rank $\operatorname{RR}(A)$, defined by Brown and Pedersen [4], is somewhat related to the stable rank but also has some different properties. There are some large classes of $C^{*}$-algebras with real rank zero (see, e.g., [5, Section V.7]). Both the stable rank and the real rank (in particular, the condition $\operatorname{RR}(A)=0$ ) have played a significant role in the classification theory of $C^{*}$-algebras $[14,26]$.

For unital $A$, the stable $\operatorname{rank} \operatorname{sr}(A)$ is either $\infty$ or the smallest possible integer $n$ such that each $n$-tuple in $A^{n}$ can be approximated in norm by $n$-tuples $\left(b_{1}, \ldots, b_{n}\right)$ such that $\sum_{i=1}^{n} b_{i}^{*} b_{i}$ is invertible. Similarly, the real rank $\operatorname{RR}(A)$ is either $\infty$ or the smallest non-negative integer $n$ such that each $(n+1)$-tuple of self-adjoint elements in $A^{n+1}$ can be approximated in norm by $(n+1)$-tuples $\left(b_{0}, b_{1}, \ldots, b_{n}\right)$ of self-adjoint elements such that $\sum_{i=0}^{n} b_{i}^{2}$ is invertible. For non-unital $A$, these ranks are defined to be those of the unitization of $A$. For the algebra of continuous functions on a compact Hausdorff

2000 Mathematics Subject Classification: 22D15, 46L35, 54H15. rank.

Key words and phrases: transformation group, group $C^{*}$-algebra, stable rank, real

The second-named author was supported by a travel grant from the German Research Foundation. 
space $X$ one has $\operatorname{RR}(C(X))=\operatorname{dim} X$ and $\operatorname{sr}(C(X))=\lfloor(\operatorname{dim} X) / 2\rfloor+1$, where $\operatorname{dim} X$ is the covering dimension of $X$ (see [23]). Thus the real rank and the stable rank can be considered as non-commutative analogues of the real and complex dimension of topological spaces.

There is now an extensive literature on stable rank and real rank, especially for group $C^{*}$-algebras (see $[1,6,18,29,30]$ and the references therein). It seems that less is known in general about transformation group $C^{*}$-algebras $C_{0}(X) \rtimes G$, although some important and difficult examples have been calculated. For example, it is known that irrational rotation algebras and Bunce-Deddens algebras have stable rank 1 and real rank 0 (see [5] and the references therein). On the other hand, it has been claimed that if $G$ is a second countable, connected compact group, and $X$ is a second countable locally compact Hausdorff space, then $\operatorname{RR}\left(C_{0}(X) \rtimes G\right) \leq \operatorname{dim}(X / G)$ [21, Theorem 2.1] and $\operatorname{sr}\left(C_{0}(X) \rtimes G\right) \leq 1+\lfloor\operatorname{dim}(X / G) / 2\rfloor[21$, Proposition 2.3]. However, as we shall point out at the end of Section 1, the proofs in [21] contain serious errors. Nevertheless, the above inequality for the stable rank follows from [27, Theorem 3.4 and Proposition 3.1] provided that $G$ is a compact Lie group and $X$ is locally of finite $G$-orbit type.

In this paper, we establish explicit formulae for the stable rank and the real rank of $C_{0}(X) \rtimes G$ when $G$ is a second countable compact group, $X$ is a second countable locally compact Hausdorff space and $X$ is locally of finite $G$-orbit type (Theorems 2.3 and 2.4). Let $\mathcal{H}$ be a representative system of the conjugacy classes of stability groups for the action of $G$ on $X$ and, for $H \in \mathcal{H}$, let $X_{H}$ denote the set of points in $X$ with stability group equal to $H$ and let $N_{G}(H)=\left\{g \in G: g^{-1} H g=H\right\}$, the normaliser of $H$ in $G$. We then show, for example, that $\operatorname{RR}\left(C_{0}(X) \rtimes G\right)=0$ if and only if $\operatorname{dim}(X / G)=0$, and if $1 \leq \operatorname{dim}(X / G)<\infty$, then

$$
\operatorname{RR}\left(C_{0}(X) \rtimes G\right)=\max \left\{1, \max _{H \in \mathcal{H}}\left\{\left\lceil\frac{\operatorname{dim}\left(X_{H} / N_{G}(H)\right)}{2[G: H]-1}\right\rceil\right\}\right\},
$$

where $\frac{d}{\infty}$ is understood as 0 . In Theorem 3.1 we apply the results of Section 2 to determine the ranks of $C_{0}\left(\mathbb{R}^{n}\right) \rtimes G$, where $G$ is a connected closed subgroup of $\mathrm{SO}(n)$ acting on $\mathbb{R}^{n}$ by rotation.

The authors are grateful to the referee for helpful comments which have led to a number of clarifications.

\section{Preliminaries: transformation groups and covering dimen-} sion. Let $(G, X)$ be a topological transformation group. That is, $G$ is a topological group and $X$ is a Hausdorff space together with a continuous map $G \times X \rightarrow X,(g, x) \mapsto g \cdot x$, such that $e \cdot x=x$ and $h \cdot(g \cdot x)=(h g) \cdot x$ for all $g, h \in G$ and $x \in X$. For $x \in X$, let $S_{x}$ denote the stabiliser of $x$, that is, $S_{x}=\{g \in G: g \cdot x=x\}$, and let $G \cdot x=\{g \cdot x: g \in G\}$ denote 
the orbit of $x$. The quotient space $X / G$ is always endowed with the quotient topology. Note that the quotient map $X \rightarrow X / G$ is continuous and open. If $G$ is compact then the quotient map is also closed and proper, and $X / G$ is Hausdorff [3]. The transformation group $(G, X)$ is said to be locally compact if both $G$ and $X$ are locally compact.

A subgroup $S$ of $G$ is called a principal stability group if there exists a dense subset $D$ of $X$ such that $S_{x}$ is conjugate to $S$ for every $x \in D$. The space $X$ is of finite $G$-orbit type if there exist subgroups $H_{1}, \ldots, H_{n}$ of $G$ such that for each $x \in X, S_{x}$ is conjugate to one of the $H_{j}$. Moreover, $X$ is said to be locally of finite $G$-orbit type if every point in $X$ has a ( $G$-invariant) neighbourhood which is of finite $G$-orbit type. If $G$ is a compact Lie group and $X$ is a topological manifold, then $X$ is locally of finite $G$-orbit type [3, Remark after IV.1.2], and if in addition $X / G$ is connected, then there exists a principal stability group [15].

For every closed subgroup $H$ of $G$, let $X_{H}=\left\{x \in X: S_{x}=H\right\}$. We shall several times use the fact that if $G$ is compact and $g^{-1} H g \subseteq H$ for some $g \in G$, then actually $g^{-1} H g=H$ so that $g \in N_{G}(H)$. This can be seen as follows. The closed subsemigroup of $G$ generated by $g$ is already a group (see $[11,(9.28)(\mathrm{a})])$. Hence there exists a sequence $\left(n_{j}\right)_{j}$ of natural numbers such that $g^{n_{j}} \rightarrow e$. Since

for all $n \in \mathbb{N}$, it follows that $g^{-1} H g=H$.

$$
g^{-n} H g^{n} \subseteq g^{-n+1} H g^{n-1} \subseteq \cdots \subseteq H
$$

Lemma 1.1. Let $(G, X)$ be a topological transformation group where $G$ is compact and $X$ is of finite $G$-orbit type. Then, for some stability group $S$, the set $\left\{x \in X: S_{x}\right.$ is conjugate to $\left.S\right\}$ is open in $X$.

Proof. Let $H_{1}, \ldots, H_{n}$ be representatives of the different conjugacy classes of stability groups. Towards a contradiction, assume that for each $1 \leq$ $k \leq n$, there exist $j_{k} \in\{1, \ldots, n\}$ and $h_{k} \in G$ such that $j_{k} \neq k$ and $h_{k} H_{j_{k}} h_{k}^{-1} \subseteq H_{k}$. Then, with $j_{0}=1$, we find sequences $\left(j_{k}\right)_{k} \subseteq\{1, \ldots, n\}$ and $\left(g_{k}\right)_{k} \subseteq G$ such that $j_{k} \neq j_{k-1}$ and

$$
H_{j_{0}} \supseteq g_{1} H_{j_{1}} g_{1}^{-1} \supseteq \cdots \supseteq g_{k} H_{j_{k}} g_{k}^{-1} \supseteq \cdots
$$

for all $k \in \mathbb{N}$. There must exist $l, k \in\{1, \ldots, n\}$ with $l<k$ and $j_{l}=j_{k}$. Then $H_{j_{l}} \supseteq g_{l}^{-1} g_{k} H_{j_{l}} g_{k}^{-1} g_{l}^{-1}$, which in turn implies that $g_{l} H_{j_{l}} g_{l}^{-1}=g_{k} H_{j_{k}} g_{k}^{-1}$. Since $j_{k} \neq j_{k-1}$, we have $l<k-1$ and hence

$$
g_{l} H_{j_{l}} g_{l}^{-1} \supseteq g_{k-1} H_{j_{k-1}} g_{k-1}^{-1} \supseteq g_{k} H_{j_{k}} g_{k}^{-1} .
$$

This shows that $H_{j_{k}}$ and $H_{j_{k-1}}$ are conjugate, a contradiction.

Thus $H_{1}$, say, has the property that $g H_{j} g^{-1} \nsubseteq H_{1}$ for any $2 \leq j \leq n$ and $g \in G$. Let

$$
C=\left\{x \in X: S_{x} \text { contains a conjugate of } H_{j} \text { for some } 2 \leq j \leq n\right\} .
$$


Then $C$ is closed in $X$. Indeed, let $\left(x_{\lambda}\right)_{\lambda}$ be a net in $C$ converging to some $x \in X$ and let $g_{\lambda} \in G$ and $j_{\lambda} \in\{2, \ldots, n\}$ be such that $S_{x_{\lambda}} \supseteq g_{\lambda} H_{j_{\lambda}} g_{\lambda}^{-1}$. After passing to a subnet if necessary, we can assume that $j_{\lambda}=j$ for all $\lambda$ and $g_{\lambda} \rightarrow g$ for some $g \in G$. It follows that $g H_{j} g^{-1} \subseteq S_{x}$, whence $x \in C$.

Finally, observe that

$$
X \backslash C=\left\{x \in X: S_{x} \text { is conjugate to } H_{1}\right\} .
$$

Clearly, if $x \in X \backslash C$, then $S_{x}$ is not conjugate to any of $H_{2}, \ldots, H_{n}$ and hence must be conjugate to $H_{1}$. On the other hand, if $S_{x}$ is conjugate to $H_{1}$ then, for each $j \geq 2, S_{x}$ cannot contain a conjugate of $H_{j}$ because $H_{1}$ does not.

Of course, in the setting of Lemma 1.1, if there exists a principal stability group, then the subgroup $S$ must be one. Note, however, that a principal stability group need not exist when $X$ is of finite $G$-orbit type. A simple example is provided by $G=\mathrm{SO}(n) \times \mathrm{SO}(m)$ acting by rotation on $X=$ $\left(\mathbb{R}^{n} \times\{0\}\right) \cup\left(\{0\} \times \mathbb{R}^{m}\right) \subseteq \mathbb{R}^{n} \times \mathbb{R}^{m}$ for $m, n \geq 3$.

Let $\mathcal{K}(G)$ denote the set of all closed subgroups of $G$ endowed with Fell's topology [9]. Recall that a base for this topology is formed by the sets

$$
U(C, \mathcal{F})=\{H \in \mathcal{K}(G): H \cap C=\emptyset, H \cap V \neq \emptyset \text { for all } V \in \mathcal{F}\},
$$

where $C$ is a compact subset of $G$ and $\mathcal{F}$ is a finite family of non-empty open subsets of $G$. The following lemma might well be known. We include the short proof for the reader's convenience.

Lemma 1.2. Let $(G, X)$ be a topological transformation group with $G$ compact. Suppose there exists a closed subgroup $H$ of $G$ such that the stabiliser of every point in $X$ is conjugate to $H$. Then the stabiliser map $x \mapsto S_{x}$ from $X$ into $\mathcal{K}(G)$ is continuous.

Proof. It suffices to show that if $\left(x_{\lambda}\right)_{\lambda}$ is any net in $X$ converging to some $x \in X$, then for some subnet of $\left(x_{\lambda}\right)_{\lambda}$, the stability groups converge to $S_{x}$ in $\mathcal{K}(G)$. Choose $g_{\lambda} \in G$ and $g \in G$ such that $S_{x_{\lambda}}=g_{\lambda}^{-1} H g_{\lambda}$ for all $\lambda$ and $S_{x}=g H^{-1}$. Since $G$ is compact, after passing to a subnet if necessary, we can assume that $g_{\lambda} \rightarrow g_{0}$ for some $g_{0} \in G$. Then, since $H$ is compact, $g_{\lambda}^{-1} H g_{\lambda} \rightarrow g_{0}^{-1} H g_{0}$ by definition of the topology on $\mathcal{K}(G)$. On the other hand, for $h \in H,\left(g_{\lambda}^{-1} h g_{\lambda}\right) \cdot x_{\lambda}=x_{\lambda} \rightarrow x$. It follows that $\left(g_{0} g\right)^{-1} H g_{0} g \subseteq H$, and this implies that $g_{0}^{-1} H g_{0}=g H g^{-1}=S_{x}$, as was to be shown.

Similar arguments show that if $G$ has a principal stability subgroup $S$ (for example if $G$ is a closed subgroup of $\mathrm{SO}(n)$ acting on $\mathbb{R}^{n} \backslash\{0\}$ by rotation) then continuity of the stabiliser map implies that every stability subgroup is conjugate to $S$.

Corollary 1.3. Suppose that $(G, X)$ is a locally compact transformation group where $G$ is compact and $X$ is locally of finite $G$-orbit type. Then 
for any closed subgroup $H$ of $G$, the space $X_{H}$ (with the relative topology) is locally compact.

Proof. Suppose first of all that $X$ is of finite $G$-orbit type. By Lemma 1.1 there is a representative system $H_{1}, \ldots, H_{r}$ of the conjugacy classes of stability groups such that the $G$-invariant sets

$$
V_{j}=\left\{x \in X: S_{x} \text { is conjugate to } H_{j}\right\}
$$

have the property that $V_{1}$ is open in $X$ (and hence is locally compact) and for $1 \leq j \leq r-1, V_{j+1}$ is open in $X \backslash \bigcup_{k=1}^{j} V_{k}$. Since $X \backslash \bigcup_{k=1}^{j} V_{k}$ is closed in $X$, it follows that $V_{j+1}$ is locally compact. For $1 \leq j \leq r$, the stabiliser map $x \mapsto S_{x}$ is continuous on $V_{j}$ by Lemma 1.2. Therefore $X_{H_{j}}$ is closed in $V_{j}$ and hence locally compact.

For the general case, suppose that $H$ is a closed subgroup of $G$ and that $x \in X_{H}$. Let $U$ be a relatively compact, $G$-invariant open neighbourhood of $x$ in $X$. Then $U$ is of finite orbit type, and hence $X_{H} \cap U=U_{H}$ is a locally compact space. So there is a compact set $N$ which is a neighbourhood of $x$ in the space $X_{H} \cap U$. Since $U$ is open in $X, N$ is a neighbourhood of $x$ in the space $X_{H}$.

For any topological space $X$, let $\mathcal{C}(X)$ denote the set of all compact subsets of $X$. Our general reference for the covering dimension $\operatorname{dim} X$ of $X$ is $[23]$.

Lemma 1.4. Let $X$ be a locally compact Hausdorff space and $Y$ a discrete space. Let $G$ be a compact group acting on $X$ and $Y$ and hence on the product space $X \times Y$. Then

$$
\sup \{\operatorname{dim} C: C \in \mathcal{C}((X \times Y) / G)\}=\sup \{\operatorname{dim} K: K \in \mathcal{C}(X / G)\} .
$$

Proof. Since $Y$ is discrete, $(X \times Y) / G$ is a disjoint union of open subsets $(X \times G \cdot y) / G, y \in Y$. Hence, for any $C \in \mathcal{C}((X \times Y) / G)$, since $C$ meets only finitely many of the disjoint sets $(X \times G \cdot y) / G$,

$$
\operatorname{dim} C=\max \{\operatorname{dim}(C \cap(X \times G \cdot y) / G): y \in Y\} .
$$

To prove the lemma, we can therefore assume that $Y=G \cdot y$ for some $y \in Y$. Since $G$ is compact, $Y$ is finite and the normal subgroup $H$ of $G$ defined by

$$
H=\{g \in G: g \cdot z=z \text { for all } z \in Y\}
$$

has finite index in $G$. Now, since the action of $H$ on $Y$ is trivial, the spaces $(X \times Y) / H$ and $(X / H) \times Y$ are canonically homeomorphic. We conclude that, replacing $X$ with $X / H$ and $G$ with $G / H$, it suffices to prove the lemma in the case of a finite group $G$.

Let $q_{X}: X \rightarrow X / G$ and $q_{X \times Y}: X \times Y \rightarrow(X \times Y) / G$ denote the quotient maps. Both $q_{X}$ and $q_{X \times Y}$ are continuous, open, proper surjections. Now, if $K \in \mathcal{C}(X / G)$ then $q_{X}^{-1}(K)$ is a compact space and $q_{X}^{-1}(t)$ is finite for every 
$t \in X / G$. Since compact spaces are paracompact, $\operatorname{dim} K=\operatorname{dim} q_{X}^{-1}(K)$ by [23, Chapter 9, Proposition 2.16]. Let $C=q_{X \times Y}\left(q_{X}^{-1}(K) \times Y\right)$. Then using $[23,9.2 .16]$ again, we obtain

$$
\operatorname{dim} C=\operatorname{dim}\left(q_{X}^{-1}(K) \times Y\right)=\operatorname{dim}\left(q_{X}^{-1}(K)\right) .
$$

Conversely, let $C \in \mathcal{C}((X \times Y) / G)$ be given. Then $q_{X \times Y}^{-1}(C)$ is compact and hence equals $\bigcup_{j=1}^{n} X_{j} \times\left\{y_{j}\right\}$, where $y_{1}, \ldots, y_{n}$ are distinct elements of $Y$ and $X_{1}, \ldots, X_{n}$ are compact subsets of $X$. Since the sets $X \times\{y\}, y \in Y$, are disjoint and clopen,

$$
\operatorname{dim} q_{X \times Y}^{-1}(C)=\max _{1 \leq j \leq n} \operatorname{dim}\left(X_{j} \times\left\{y_{j}\right\}\right)=\operatorname{dim} X_{j_{0}}
$$

say. Let $K=q_{X}\left(X_{j_{0}}\right)$; then applying $[23,9.2 .16]$ twice more, we get

$$
\operatorname{dim} C=\operatorname{dim} q_{X \times Y}^{-1}(C)=\operatorname{dim} X_{j_{0}} \leq \operatorname{dim} q_{X}^{-1}(K)=\operatorname{dim} K,
$$

as required.

Lemma 1.5. Let $(G, X)$ be a locally compact transformation group where $G$ is compact, and let $H$ be a closed subgroup of $G$. Then

(i) $X_{H} / N_{G}(H)$ is homeomorphic to $G \cdot X_{H} / G$;

(ii) if $X / G$ is second countable and $X$ is locally of finite $G$-orbit type, then

$$
\operatorname{dim}\left(X_{H} / N_{G}(H)\right) \leq \operatorname{dim}(X / G) .
$$

Proof. (i) Define a map $\phi$ from $X_{H} / N_{G}(H)$ into $X / G$ by $\phi\left(N_{G}(H) \cdot x\right)=$ $G \cdot x$. Then $\phi$ is continuous since the quotient map $X \rightarrow X / G$ is continuous and the quotient map $X_{H} \rightarrow X_{H} / N_{G}(H)$ is open. If $x_{1}, x_{2} \in X_{H}$ are such that $x_{2}=g \cdot x_{1}$ for some $g \in G$, then

$$
H=S_{x_{2}}=g S_{x_{1}} g^{-1}=g H g^{-1}
$$

and hence $g \in N_{G}(H)$. Thus $\phi$ is injective and its range is the subspace $G \cdot X_{H} / G$ of $X / G$. To prove that $\phi: X_{H} / N_{G}(H) \rightarrow G \cdot X_{H} / G$ is open, let $U$ be an open subset of $X_{H} / N_{G}(H)$, and let $q_{1}: X_{H} \rightarrow X_{H} / N_{G}(H)$ and $q_{2}: G \cdot X_{H} \rightarrow G \cdot X_{H} / G$ denote the quotient maps. Let $x \in q_{2}^{-1}(\phi(U))$ and let $\left(x_{\alpha}\right)_{\alpha}$ be any net in $G \cdot X_{H}$ converging to $x$. For each $\alpha, x_{\alpha}=g_{\alpha} \cdot y_{\alpha}$ where $g_{\alpha} \in G, y_{\alpha} \in X_{H}$. Also, there exists $y \in q_{1}^{-1}(U) \subseteq X_{H}$ such that $q_{2}(x)=\phi\left(q_{1}(y)\right)=q_{2}(y)$, and so $x=g_{0} \cdot y$ for some $g_{0} \in G$. Passing to a subnet of $\left(g_{\alpha}\right)_{\alpha}$ and to the corresponding subnet of $\left(x_{\alpha}\right)_{\alpha}$, we may assume that $g_{\alpha} \rightarrow g_{1} \in G$. Then $y_{\alpha} \rightarrow\left(g_{1}^{-1} g_{0}\right) \cdot y$ and therefore

$$
H \subseteq S_{\left(g_{1}^{-1} g_{0}\right) \cdot y}=\left(g_{1}^{-1} g_{0}\right) S_{y}\left(g_{1}^{-1} g_{0}\right)^{-1}=\left(g_{1}^{-1} g_{0}\right) H\left(g_{1}^{-1} g_{0}\right)^{-1} .
$$

This implies that $H=\left(g_{1}^{-1} g_{0}\right) H\left(g_{1}^{-1} g_{0}\right)^{-1}$, whence $g_{1}^{-1} g_{0} \in N_{G}(H)$. Eventually $\left(g_{0}^{-1} g_{1}\right) \cdot y_{\alpha} \in q_{1}^{-1}(U)$ and so eventually $q_{2}\left(x_{\alpha}\right)=q_{2}\left(y_{\alpha}\right) \in \phi(U)$. Thus the original net $\left(x_{\alpha}\right)_{\alpha}$ is frequently in $q_{2}^{-1}(\phi(U))$. This shows that $q_{2}^{-1}(\phi(U))$ is open, and hence so is $\phi(U)$. 
(ii) Recall that a second countable, locally compact Hausdorff space $Y$ is normal [23, Chapter 1, Lemma 4.7] and every locally compact subspace $Z$ of such a space $Y$ is an $F_{\sigma}$-set in $Y$ and hence $\operatorname{dim} Z \leq \operatorname{dim} Y$ [23, Chapter 3, Corollary 6.3]. By (i) and Corollary 1.3, we obtain

$$
\operatorname{dim}\left(X_{H} / N_{G}(H)\right)=\operatorname{dim}\left(G \cdot X_{H} / G\right) \leq \operatorname{dim}(X / G),
$$

as required.

REMARK 1.6. (i) Let $X$ be a second countable locally compact Hausdorff space. Then $\operatorname{dim} X=\sup \{\operatorname{dim} C: C \in \mathcal{C}(X)\}$. Indeed, each such $C$ is closed and hence $\operatorname{dim} C \leq \operatorname{dim} X$. On the other hand, since $X$ is normal and $\sigma$ compact, $\operatorname{dim} X \leq \sup \{\operatorname{dim} C: C \in \mathcal{C}(X)\}$ by the countable sum theorem [23, Chapter 3, Theorem 2.5].

(ii) Let $X$ be a second countable locally compact Hausdorff space, and let $X_{j}, 1 \leq j \leq n$, be pairwise disjoint subsets of $X$ such that $X=\bigcup_{j=1}^{n} X_{j}$ and $\bigcup_{j=k}^{n} X_{j}$ is closed in $X$ for every $2 \leq k \leq n$. Then

$$
\operatorname{dim} X=\max \left\{\operatorname{dim} X_{j}: 1 \leq j \leq n\right\} .
$$

This follows from $n-1$ applications of the fact that

$$
\operatorname{dim} X=\max \{\operatorname{dim} Y, \operatorname{dim}(X \backslash Y)\}
$$

for any closed subset $Y$ of a second countable, locally compact Hausdorff space $X[23,3.5 .8,3.6 .7,2.2 .9]$.

Finally, as promised in the introduction, we point out the errors in the proof of [21, Theorem 2.1]. The first one is the claim that, if $F$ is the set of fixed points in $X$, then $C_{0}(X \backslash F) \rtimes G$ is isomorphic to $C_{0}((X \backslash F) / G) \otimes \mathcal{K}$, where $\mathcal{K}$ is the algebra of compact operators on a separable Hilbert space. But this would imply that $C_{0}(X \backslash F) \rtimes G$ was a continuous trace $C^{*}$-algebra, which need not be the case (see the example below). The second problem concerns the application of [21, Proposition 1.10] to the short exact sequence which begins $0 \rightarrow C_{0}(X \backslash F) \rtimes G \rightarrow C_{0}(X) \rtimes G \rightarrow \cdots$. In fact, neither $C_{0}(X \backslash F) \rtimes G$ nor $C_{0}(X) \rtimes G$ need satisfy the hypotheses of [21, Proposition 1.10]. In particular, in the example below, neither of these algebras has Hausdorff spectrum. In addition, this example illustrates the sets $X_{H}$.

EXAmple 1.7. Let $X=\mathbb{R}^{n} \times \mathbb{R}^{m}, n, m \geq 3$, and let $G=\mathrm{SO}(n) \times$ $\mathrm{SO}(m)$ act on $X$ in the obvious manner. Then $F=X_{G}=\{(0,0)\}$. Let $v=(1,0, \ldots, 0) \in \mathbb{R}^{n}$ and $w=(1,0, \ldots, 0) \in \mathbb{R}^{m}$, and embed $\operatorname{SO}(n-1)$ into $\mathrm{SO}(n)$ as the subgroup fixing $v$ and $\mathrm{SO}(m-1)$ into $\mathrm{SO}(m)$ as the subgroup fixing $w$. Then for every non-zero $x \in X$ there exist $s, t \neq 0$ such that $x$ belongs to the $G$-orbit of one of the vectors $(t v, s w),(t v, 0)$ and $(0, s w)$. Thus, apart from $G$ itself, there are three conjugacy classes of stability subgroups, with representatives 


$$
\begin{aligned}
& H_{1}=S_{(t v, s w)}=\mathrm{SO}(n-1) \times \mathrm{SO}(m-1), \\
& H_{2}=S_{(t v, 0)}=\mathrm{SO}(n-1) \times \mathrm{SO}(m), \\
& H_{3}=S_{(0, s w)}=\mathrm{SO}(n) \times \mathrm{SO}(m-1) .
\end{aligned}
$$

Then $N_{G}\left(H_{j}\right)=H_{j}$ for $1 \leq j \leq 3$ and $X_{H_{1}}=\{(t v, s w): t, s \neq 0\}$, $X_{H_{2}}=\{(t v, 0): t \neq 0\}$ and $X_{H_{3}}=\{(0, s w): s \neq 0\}$.

The fact that $S_{(t v, w)}=\mathrm{SO}(n-1) \times \mathrm{SO}(m-1)$ for all $t \neq 0$ shows that the map $x \mapsto S_{x}$ is not continuous at $(0, w)$. It follows from [7, Corollary 2] that neither $C_{0}(X \backslash F) \rtimes G$ nor $C_{0}(X) \rtimes G$ has Hausdorff spectrum.

2. The ranks of compact transformation group $C^{*}$-algebras. We note here that if $J$ is a closed ideal of a $C^{*}$-algebra $A$, then $\operatorname{sr}(J), \operatorname{sr}(A / J) \leq$ $\operatorname{sr}(A)[24$, Section 4] and similarly for the real rank [8, Théorème 1.4]. Also, recall that, for a non-negative real number $t,\lfloor t\rfloor$ denotes the greatest integer $n$ such that $n \leq t$ and $\lceil t\rceil$ is the least integer $m$ such that $m \geq t$.

Let $(G, X)$ be a locally compact transformation group and let $C_{0}(X) \rtimes G$ denote the associated crossed product $C^{*}$-algebra. We shall frequently use the fact that if $U$ is an open $G$-invariant subset of $X$ then $C_{0}(U) \rtimes G$ embeds in $C_{0}(X) \rtimes G$ as a closed ideal with quotient canonically isomorphic to $C_{0}(X \backslash U) \rtimes G$. This is a well known consequence of the universal property of (full) crossed products (see, for example, [13]).

For $x \in X$ and a representation $\tau$ of $S_{x}$ in the Hilbert space $\mathcal{H}(\tau)$, let $\pi_{x, \tau}$ be the $*$-representation of $C_{0}(X)$ in $\mathcal{H}(\tau)$ defined by $\pi_{x, \tau}(f)=f(x) \operatorname{id}_{\mathcal{H}(\tau)}$, $f \in C_{0}(X)$. Then $\left(\pi_{x, \tau}, \tau\right)$ is a covariant representation of $\left(C_{0}(X), S_{x}, \alpha\right)$, where $\alpha$ denotes the action of $G$ on $C_{0}(X)$. If $\tau$ is irreducible, then the induced representation $\operatorname{ind}_{S_{x}}^{G}\left(\pi_{x, \tau}, \tau\right)$ of $C_{0}(X) \rtimes G$ is irreducible. If $\sigma$ and $\tau$ are two irreducible representations of $S_{x}$, then $\operatorname{ind}_{S_{x}}^{G}\left(\pi_{x, \sigma}, \sigma\right)$ and $\operatorname{ind}_{S_{x}}^{G}\left(\pi_{x, \tau}, \tau\right)$ are equivalent if and only if $\sigma$ and $\tau$ are equivalent. Moreover, if $G$ is compact, then every irreducible representation of $C_{0}(X) \rtimes G$ is obtained in this way as an induced representation.

Proposition 2.1. Let $(G, X)$ be a transformation group, where $X$ is a locally compact Hausdorff space and $G$ is a compact group. Suppose that there exists a subgroup $H$ of $G$ such that the stabiliser of every point in $X$ is conjugate to $H$.

(a) Suppose that $H$ has finite index in $G$. Then

(i) $\operatorname{sr}\left(C_{0}(X) \rtimes G\right)$

$$
=\sup \left\{1+\left\lceil\frac{1}{[G: H]}\left\lfloor\frac{1}{2} \operatorname{dim} C\right\rfloor\right]: C \in \mathcal{C}\left(X_{H} / N_{G}(H)\right)\right\} ;
$$

(ii) $\operatorname{RR}\left(C_{0}(X) \rtimes G\right)=\sup \left\{\left\lceil\frac{\operatorname{dim} C}{2[G: H]-1}\right\rceil: C \in \mathcal{C}\left(X_{H} / N_{G}(H)\right)\right\}$. 
Suppose, in addition, that $X$ is second countable. Then

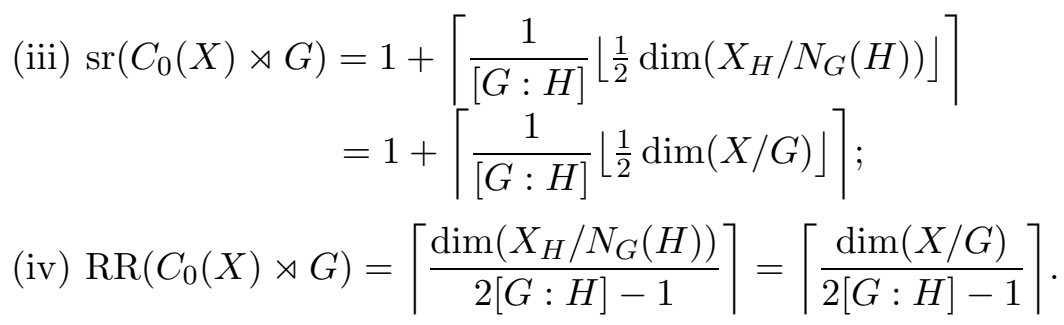

(b) Suppose that $H$ has infinite index in $G$ and that both $X$ and $G$ are second countable and $\operatorname{dim}(X / G)<\infty$. Then

(i) $\operatorname{sr}\left(C_{0}(X) \rtimes G\right)=\min \left\{2,1+\left\lfloor\frac{1}{2} \operatorname{dim}\left(X_{H} / N_{G}(H)\right)\right\rfloor\right\}$

$$
=\min \left\{2,1+\left\lfloor\frac{1}{2} \operatorname{dim}(X / G)\right\rfloor\right\}
$$

(ii) $\operatorname{RR}\left(C_{0}(X) \rtimes G\right)=\min \left\{1, \operatorname{dim}\left(X_{H} / N_{G}(H)\right)\right\}$

$$
=\min \{1, \operatorname{dim}(X / G)\} \text {. }
$$

Proof. Let $A=C_{0}(X) \rtimes G$ and note that, since the stabiliser map $x \mapsto S_{x}$ from $X$ into $\mathcal{K}(G)$ is continuous (Lemma 1.2) and $G$ is compact, $A$ has continuous trace [7, Corollary 2]. By hypothesis, for each $x \in X$ there exists $g_{x} \in G$ such that $S_{x}=g_{x} H g_{x}^{-1}$. Thus there is a well defined $G$-equivariant map $\pi$ from $X$ to the left coset space $G / N_{G}(H)$ given by $\pi(x)=g_{x} N_{G}(H)$. Clearly, $\pi^{-1}(\{e H\})=X_{H}$. An argument similar to (but simpler than) the proof of Lemma 1.2 shows that $\pi$ is continuous. Hence, by [10, Theorem 17] the two $C^{*}$-algebras $A$ and $C_{0}\left(X_{H}\right) \rtimes N_{G}(H)$ are Morita equivalent and hence their spectra are homeomorphic. On the other hand, since $\left(N_{G}(H), X_{H}\right)$ is a compact transformation group for which all of the stability subgroups are equal to $H$, it follows from [32, Corollary 5.12] that the continuous trace $C^{*}$-algebra $C_{0}\left(X_{H}\right) \rtimes N_{G}(H)$ has spectrum homeomorphic to the quotient space $\left(X_{H} \times \widehat{H}\right) / N_{G}(H)$. Indeed, elements of $X_{H} \times \widehat{H}$ induce irreducible representations of $C_{0}\left(X_{H}\right) \rtimes N_{G}(H)$ as described above, and for $g \in N_{G}(H), x \in X$ and $\tau \in \widehat{H}$, the representation induced from $(x, \tau)$ is equivalent to the one induced from $g \cdot(x, \tau)=(g \cdot x, g \cdot \tau)$.

Assume first that $G, H$ and $X$ satisfy the hypotheses of (b). Then, since $X$ and $G$ are second countable, $A$ is separable and $\operatorname{dim} \pi=\aleph_{0}$ for every $\pi \in \widehat{A}$. Moreover, $A$ has continuous trace. By Lemmas 1.4 and 1.5 , for every compact subset $C$ of $\left(X_{H} \times \widehat{H}\right) / N_{G}(H)$,

$$
\begin{aligned}
\operatorname{dim} C & \leq \sup \left\{\operatorname{dim} K: K \in \mathcal{C}\left(X_{H} / N_{G}(H)\right)\right\} \\
& \leq \operatorname{dim}\left(X_{H} / N_{G}(H)\right) \leq \operatorname{dim}(X / G) .
\end{aligned}
$$

Since $\widehat{A}$ is a second countable, locally compact Hausdorff space and is homeomorphic to $\left(X_{H} \times \widehat{H}\right) / N_{G}(H)$, Lemmas 1.4 and 1.5 and Remark 1.6(i) show that $\operatorname{dim} \widehat{A} \leq \operatorname{dim}(X / G)<\infty$. The following formulae are given in [1, 
Proposition 1.2(b)]:

$$
\begin{aligned}
\operatorname{RR}(A) & =\min \{1, \sup \{\operatorname{dim} C: C \in \mathcal{C}(\widehat{A})\}\} \\
\operatorname{sr}(A) & =\min \left\{2,1+\left\lfloor\frac{1}{2} \sup \{\operatorname{dim} C: C \in \mathcal{C}(\widehat{A})\}\right\rfloor\right\} .
\end{aligned}
$$

The statements in (b) now follow from Lemmas 1.4 and 1.5 together with Remark 1.6(i) again.

Now suppose that $H$ has finite index in $G$. For $d \in \mathbb{N}$, define $\widehat{H}_{d}=$ $\{\tau \in \widehat{H}: \operatorname{dim} \tau=d\}$. Then $X_{H} \times \widehat{H}$ is the disjoint union of the open and closed sets $X_{H} \times \widehat{H}_{d}, d \in \mathbb{N}$, and each $X_{H} \times \widehat{H}_{d}$ is $N_{G}(H)$-invariant. It therefore follows that $\left(X_{H} \times \widehat{H}\right) / N_{G}(H)$ is the disjoint union of open and closed sets $\left(X_{H} \times \widehat{H}_{d}\right) / N_{G}(H), d \in \mathbb{N}$.

Let $A_{d}$ denote the closed ideal of $A$ with $\widehat{A}_{d}=\left(X_{H} \times \widehat{H}_{d}\right) / N_{G}(H)$. Note that points of $\left(X_{H} \times \widehat{H}_{d}\right) / N_{G}(H)$ give rise to irreducible representations of $A$, with dimension $d[G: H]$, by the induction process described at the start of this section. Hence each $A_{d}$ is $d[G: H]$-homogeneous. Since the sets $\widehat{A}_{d}$ are open and closed, $A$ is isomorphic to the $c_{0}$-direct sum of the $A_{d}$. Thus

$$
\operatorname{RR}(A)=\sup _{d \in \mathbb{N}}\left\{\operatorname{RR}\left(A_{d}\right)\right\}, \quad \operatorname{sr}(A)=\sup _{d \in \mathbb{N}}\left\{\operatorname{sr}\left(A_{d}\right)\right\} .
$$

Since $A_{d}$ is $d[G: H]$-homogeneous, the formulae in [1, Proposition 1.2(a)] for the real and stable ranks of an $n$-homogeneous $C^{*}$-algebra give

$$
\begin{aligned}
\operatorname{RR}\left(A_{d}\right) & =\sup \left\{\left\lceil\frac{\operatorname{dim} C}{2 d[G: H]-1}\right\rceil: C \in \mathcal{C}\left(\widehat{A}_{d}\right)\right\}, \\
\operatorname{sr}\left(A_{d}\right) & =\sup \left\{1+\left\lceil\frac{1}{d[G: H]}\left\lfloor\frac{1}{2} \operatorname{dim} C\right]\right\rceil: C \in \mathcal{C}\left(\widehat{A}_{d}\right)\right\} .
\end{aligned}
$$

Since $\widehat{H}$ is discrete and $N_{G}(H)$ is compact, for every $d \in \mathbb{N}$ with $\widehat{H}_{d} \neq \emptyset$,

$$
\sup \left\{\operatorname{dim} C: C \in \mathcal{C}\left(\widehat{A}_{d}\right)\right\}=\sup \left\{\operatorname{dim} K: K \in \mathcal{C}\left(X_{H} / N_{G}(H)\right)\right\}
$$

by Lemma 1.4. The formulae in (a)(i),(ii) follow by taking $d=1$, and the formulae in (iii) and (iv) follow from Remark 1.6(i) and Lemma 1.5(i).

The following simple lemma, together with the countable sum theorem for the covering dimension, will allow us to obtain results in Theorems 2.3 and 2.4 when $X$ is only locally of finite $G$-orbit type.

Lemma 2.2. Let $(G, X)$ be a transformation group, where $G$ is a compact group and $X$ is a locally compact Hausdorff space. Then

(i) $\operatorname{sr}\left(C_{0}(X) \rtimes G\right)=\sup \{\operatorname{sr}(C(G \cdot K) \rtimes G): K \in \mathcal{C}(X)\}$;

(ii) $\operatorname{RR}\left(C_{0}(X) \rtimes G\right)=\sup \{\operatorname{RR}(C(G \cdot K) \rtimes G): K \in \mathcal{C}(X)\}$.

Proof. Let $A=C_{0}(X) \rtimes G$ and let $\mathcal{V}$ denote the set of all relatively compact open subsets of $X$, directed by inclusion. Then $A$ is the inductive 
limit of the family of ideals $C_{0}(G \cdot V) \rtimes G, V \in \mathcal{V}$, and hence

$$
\operatorname{sr}(A) \leq \sup _{V \in \mathcal{V}}\left\{\operatorname{sr}\left(C_{0}(G \cdot V) \rtimes G\right)\right\}, \quad \operatorname{RR}(A) \leq \sup _{V \in \mathcal{V}}\left\{\operatorname{RR}\left(C_{0}(G \cdot V) \rtimes G\right)\right\}
$$

(compare [24, Theorem 5.1] and [12, Lemma 4.1(i)]). On the other hand, $C_{0}(G \cdot V) \rtimes G$ is an ideal of $C(G \cdot \bar{V}) \rtimes G$, and for every $K \in \mathcal{C}(X), C(G \cdot K) \rtimes G$ is a quotient of $A$. It follows that

$$
\begin{aligned}
\operatorname{sr}(A) & \leq \sup _{V \in \mathcal{V}}\left\{\operatorname{sr}\left(C_{0}(G \cdot V) \rtimes G\right)\right\} \leq \sup _{V \in \mathcal{V}}\{\operatorname{sr}(C(G \cdot \bar{V}) \rtimes G)\} \\
& \leq \sup _{K \in \mathcal{C}(X)}\{\operatorname{sr}(C(G \cdot K) \rtimes G)\} \leq \operatorname{sr}(A),
\end{aligned}
$$

and similarly for the real rank.

In the following two theorems, $\mathcal{H}$ denotes a representative system of the conjugacy classes of stability groups, and $\mathcal{H}_{\text {fin }}=\{H \in \mathcal{H}:[G: H]<\infty\}$.

TheOREM 2.3. Let $(G, X)$ be a transformation group, where $X$ is a locally compact Hausdorff space, $G$ is a compact group and every stability group has finite index in $G$.

(a) Suppose that $X$ is of finite $G$-orbit type. Then

(i) $\operatorname{sr}\left(C_{0}(X) \rtimes G\right)$

$$
=\max _{H \in \mathcal{H}} \sup \left\{1+\left\lceil\frac{1}{[G: H]}\left\lfloor\frac{1}{2} \operatorname{dim} C\right\rfloor\right\rceil: C \in \mathcal{C}\left(X_{H} / N_{G}(H)\right)\right\} ;
$$

(ii) $\operatorname{RR}\left(C_{0}(X) \rtimes G\right)$

$$
=\max _{H \in \mathcal{H}} \sup \left\{\left\lceil\frac{\operatorname{dim} C}{2[G: H]-1}\right\rceil: C \in \mathcal{C}\left(X_{H} / N_{G}(H)\right)\right\} .
$$

(b) Suppose that $X$ is second countable and locally of finite $G$-orbit type. Then

(i) $\operatorname{sr}\left(C_{0}(X) \rtimes G\right)=\sup _{H \in \mathcal{H}}\left\{1+\left\lceil\frac{1}{[G: H]}\left\lfloor\frac{1}{2} \operatorname{dim}\left(X_{H} / N_{G}(H)\right)\right\rfloor\right]\right.$;

(ii) $\operatorname{RR}\left(C_{0}(X) \rtimes G\right)=\sup _{H \in \mathcal{H}}\left\{\left\lceil\frac{\operatorname{dim}\left(X_{H} / N_{G}(H)\right)}{2[G: H]-1}\right\rceil\right\}$.

Proof. (a) Let $A=C_{0}(X) \rtimes G$. To prove (i), we proceed by induction on the number of elements in $\mathcal{H}$. Notice first that by Lemma 1.1 there exists $H_{0} \in \mathcal{H}_{\text {fin }}$ such that the set $U=\left\{x \in X: S_{x}\right.$ is conjugate to $\left.H_{0}\right\}$ is open in $X$. Then, as in the proof of Proposition 2.1(a), the ideal $I=C_{0}(U) \rtimes G$ has continuous trace and is isomorphic to a $c_{0}$-direct sum of homogeneous $C^{*}$ algebras. It follows by $[20$, Lemma $5(\mathrm{a})]$ that $\operatorname{sr}(A)=\max \{\operatorname{sr}(I), \operatorname{sr}(A / I)\}$.

Now $\operatorname{sr}(I)$ is given by formula (a)(ii) of Proposition 2.1. Since $\mathcal{H} \backslash\left\{H_{0}\right\}$ represents the conjugacy classes of stability groups for the action of $G$ on $X \backslash U$, the inductive hypothesis together with $\operatorname{sr}(A)=\max \{\operatorname{sr}(I), \operatorname{sr}(A / I)\}$ yields the formula in (i). 
The argument for part (ii) is very similar, but requires the formula $\mathrm{RR}(A)=\max \{\operatorname{RR}(I), \operatorname{RR}(A / I)\}$ where the ideal $I$ is as above. The proof of this formula proceeds as in the case of stable rank [20, Lemma 5(a)], but uses the fact that the real rank does not increase on passing to a closed ideal and replaces the application of [28, Proposition 3.15] by [21, Lemma 1.9]. (Note that in the proof of [21, Lemma 1.9] there is sufficient surjectivity for the argument of [21, Proposition 1.3] to be satisfactorily applied. A full proof of [21, Proposition 1.3] has been given in [19, Proposition 1.6].)

(b) Since $X$ is a $\sigma$-compact, locally compact Hausdorff space, there exists a sequence $\left(Y_{n}\right)_{n}$ of $G$-invariant compact subsets $Y_{n}$ of $X$ such that every $G$-invariant compact subset $K$ of $X$ is contained in some $Y_{n}$. Then $C(K) \rtimes G$ is a quotient of $C\left(Y_{n}\right) \rtimes G$, and hence Lemma 2.2 shows that

$$
\begin{aligned}
\operatorname{sr}\left(C_{0}(X) \rtimes G\right) & =\sup _{n \in \mathbb{N}}\left\{\operatorname{sr}\left(C\left(Y_{n}\right) \rtimes G\right)\right\}, \\
\operatorname{RR}\left(C_{0}(X) \rtimes G\right) & =\sup _{n \in \mathbb{N}}\left\{\operatorname{RR}\left(C\left(Y_{n}\right) \rtimes G\right)\right\} .
\end{aligned}
$$

On the other hand, for any $H \in \mathcal{H}$,

$$
X_{H} / N_{G}(H)=\bigcup_{n \in \mathbb{N}}\left(Y_{n}\right)_{H} / N_{G}(H) .
$$

Furthermore, each $\left(Y_{n}\right)_{H}$ is relatively closed in $X_{H}$ and the quotient map is closed. By Corollary 1.3, $X_{H} / N_{G}(H)$ is a second countable, locally compact Hausdorff space and hence is normal. By the countable sum theorem

$$
\operatorname{dim}\left(X_{H} / N_{G}(H)\right)=\sup _{n \in \mathbb{N}}\left\{\operatorname{dim}\left(\left(Y_{n}\right)_{H} / N_{G}(H)\right)\right\} .
$$

The results now follow by applying part (a) to each $Y_{n}$ and noting that $\operatorname{dim}\left(\left(Y_{n}\right)_{H} / N_{G}(H)\right)$ is the supremum of the dimensions of its compact subsets (Remark 1.6(i)).

Theorem 2.4. Let $(G, X)$ be a transformation group, where $X$ is a second countable, locally compact Hausdorff space and $G$ is a second countable compact group. In addition, suppose that $\operatorname{dim}(X / G)<\infty$, that $X$ is locally of finite $G$-orbit type and that $\mathcal{H}_{\text {fin }} \neq \mathcal{H}$. Then

(i) $\operatorname{sr}\left(C_{0}(X) \rtimes G\right)=1$ if and only if $\operatorname{dim}(X / G) \leq 1$ and otherwise

$$
\begin{aligned}
& \operatorname{sr}\left(C_{0}(X) \rtimes G\right) \\
& =\max \left\{2, \max _{H \in \mathcal{H}_{\text {fin }}}\left\{1+\left\lceil\frac{1}{[G: H]}\left\lfloor\frac{1}{2} \operatorname{dim}\left(X_{H} / N_{G}(H)\right)\right\rfloor\right\rceil\right\}\right\},
\end{aligned}
$$

where the right hand side is interpreted as 2 if $\mathcal{H}_{\mathrm{fin}}=\emptyset$;

(ii) $\operatorname{RR}\left(C_{0}(X) \rtimes G\right)=0$ if and only if $\operatorname{dim}(X / G)=0$ and otherwise

$$
\operatorname{RR}\left(C_{0}(X) \rtimes G\right)=\max \left\{1, \max _{H \in \mathcal{H}_{\text {fin }}}\left\{\left\lceil\frac{\operatorname{dim}\left(X_{H} / N_{G}(H)\right)}{2[G: H]-1}\right\rceil\right\}\right\},
$$

where the right hand side is interpreted as 1 if $\mathcal{H}_{\mathrm{fin}}=\emptyset$. 
Proof. Since $X$ is a $\sigma$-compact locally compact Hausdorff space, there exists a sequence $\left(Y_{n}\right)_{n}$ of $G$-invariant compact subsets $Y_{n}$ of $X$ such that every $G$-invariant compact subset $K$ of $X$ is contained in some $Y_{n}$. As in the proof of Theorem 2.3(b), we obtain the equations (1), (2) and (3) above. Furthermore, by the countable sum theorem, $\operatorname{dim}(X / G)=\sup _{n \in \mathbb{N}}\left\{\operatorname{dim}\left(Y_{n} / G\right)\right\}$. Therefore, to prove the formulae of the theorem, we can assume that $X$ is compact and hence of finite $G$-orbit type.

Let $N$ be a closed normal subgroup of $G$ of finite index in $G$ such that $N \subseteq H$ for all $H \in \mathcal{H}_{\text {fin }}$. Then the set

$$
X_{\text {fin }}=\left\{x \in X:\left[G: S_{x}\right]<\infty\right\}=\left\{x \in X: S_{x} \supseteq N\right\}
$$

is closed in $X$, and $X \backslash X_{\text {fin }}$ is a non-empty, open, $G$-invariant set. Applying Lemma 1.1 to $X \backslash X_{\text {fin }}$, we conclude that there exists $S \in \mathcal{H} \backslash \mathcal{H}_{\text {fin }}$ such that the set $U=\left\{x \in X: S_{x}\right.$ is conjugate to $\left.S\right\}$ is open in $X$. Let $A=C_{0}(X) \rtimes G$ and $I=C_{0}(U) \rtimes G$, and let $\mathcal{V}$ be the collection of all $G$-invariant, relatively compact, open subsets $V$ of $U$ such that $\bar{V} \subseteq U$, directed by inclusion. To each $V \in \mathcal{V}$ associate the ideals $I_{V}=C_{0}(V) \rtimes G$ and $J_{V}=C_{0}(X \backslash \bar{V}) \rtimes G$. Then $I_{V} \cdot J_{V}=\{0\}$ for each $V$, and since $\bigcup\left\{C_{0}(V): V \in \mathcal{V}\right\}$ is dense in $C_{0}(U)$, it follows by standard arguments that $I=\bar{\bigcup}\left\{I_{V}: V \in \mathcal{V}\right\}$. Then

$$
\operatorname{sr}(A)=\max \left\{\operatorname{sr}(A / I), \sup _{V \in \mathcal{V}}\left\{\operatorname{sr}\left(A / J_{V}\right)\right\}\right\}
$$

by applying [28, Proposition 3.15] to the unitization of $A$, and

$$
\mathrm{RR}(A)=\max \left\{\operatorname{RR}(A / I), \sup _{V \in \mathcal{V}}\left\{\operatorname{RR}\left(A / J_{V}\right)\right\}\right\}
$$

by [21, Lemma 1.9] (see the remarks in the proof of Theorem 2.3(a)).

Since $A / J_{V}$ is isomorphic to $C(\bar{V}) \rtimes G$, we obtain

$$
\begin{aligned}
\operatorname{sr}(A) & =\max \left\{\operatorname{sr}(A / I), \sup _{V \in \mathcal{V}}\{\operatorname{sr}(C(\bar{V}) \rtimes G)\}\right\}, \\
\operatorname{RR}(A) & =\max \left\{\operatorname{RR}(A / I), \sup _{V \in \mathcal{V}}\{\operatorname{RR}(C(\bar{V}) \rtimes G)\}\right\} .
\end{aligned}
$$

Since $U$ is a countable union of sets $\bar{V}, X_{S}=U_{S}$ is a countable union of relatively closed sets $\bar{V} \cap X_{S}=\bar{V}_{S}$, and hence the normal space $X_{S} / N_{G}(S)$ (see Corollary 1.3) is a countable union of closed subsets $\bar{V}_{S} / N_{G}(S)$. Proposition 2.1(b)(i) and the countable sum theorem then imply that

$$
\begin{aligned}
\operatorname{sr}(A) & =\max \left\{\operatorname{sr}(A / I), \sup _{V \in \mathcal{V}}\left\{\min \left\{2,1+\left\lfloor\frac{1}{2} \operatorname{dim}\left(\bar{V}_{S} / N_{G}(S)\right)\right\rfloor\right\}\right\}\right\} \\
& =\max \left\{\operatorname{sr}(A / I), \min \left\{2,1+\sup _{V \in \mathcal{V}}\left\{\left\lfloor\frac{1}{2} \operatorname{dim}\left(\bar{V}_{S} / N_{G}(S)\right)\right\rfloor\right\}\right\}\right\} \\
& =\max \left\{\operatorname{sr}(A / I), \min \left\{2,1+\left\lfloor\frac{1}{2} \operatorname{dim}\left(X_{S} / N_{G}(S)\right)\right\rfloor\right\}\right\} .
\end{aligned}
$$

Similarly, using Proposition 2.1(b)(ii), we obtain

$$
\operatorname{RR}(A)=\max \left\{\operatorname{RR}(A / I), \min \left\{1, \operatorname{dim}\left(X_{S} / N_{G}(S)\right)\right\}\right\} .
$$


Proceeding inductively, we find that $\operatorname{sr}(A)$ is the maximum of $\operatorname{sr}\left(C\left(X_{\mathrm{fin}}\right) \rtimes G\right)$ and

$$
\max _{H \in \mathcal{H} \backslash \mathcal{H}_{\text {fin }}}\left\{\min \left\{2,1+\left\lfloor\frac{1}{2} \operatorname{dim}\left(X_{H} / N_{G}(H)\right)\right\rfloor\right\}\right\} .
$$

Similarly, $\operatorname{RR}(A)$ is the maximum of $\operatorname{RR}\left(C\left(X_{\text {fin }}\right) \rtimes G\right)$ and

$$
\max _{H \in \mathcal{H} \backslash \mathcal{H}_{\text {fin }}}\left\{\min \left\{1, \operatorname{dim}\left(X_{H} / N_{G}(H)\right)\right\}\right\} .
$$

Now, by Theorem 2.3,

$$
\begin{aligned}
\operatorname{sr}\left(C\left(X_{\text {fin }}\right) \rtimes G\right) & =\max _{H \in \mathcal{H}_{\text {fin }}}\left\{1+\left\lceil\frac{1}{[G: H]}\left\lfloor\frac{1}{2} \operatorname{dim}\left(X_{H} / N_{G}(H)\right)\right\rfloor\right\rceil,\right. \\
\operatorname{RR}\left(C\left(X_{\text {fin }}\right) \rtimes G\right) & =\max _{H \in \mathcal{H}_{\text {fin }}}\left\{\left\lceil\frac{\operatorname{dim}\left(X_{H} / N_{G}(H)\right)}{2[G: H]-1}\right\rceil\right\} .
\end{aligned}
$$

Recall that, by Lemma 1.5 and Remark 1.6(ii),

$$
\operatorname{dim}(X / G)=\max _{H \in \mathcal{H}}\left\{\operatorname{dim}\left(X_{H} / N_{G}(H)\right)\right\} .
$$

The above formulae then immediately show that $\operatorname{sr}(A)=1$ if and only if $\operatorname{dim}(X / G) \leq 1$, and $\operatorname{RR}(A)=0$ if and only if $\operatorname{dim}(X / G)=0$.

Now, assume that $\operatorname{dim}(X / G) \geq 2$, so that $\operatorname{dim}\left(X_{S} / N_{G}(S)\right) \geq 2$ for at least one $S \in \mathcal{H}$. If this happens for some $S \in \mathcal{H} \backslash \mathcal{H}_{\text {fin }}$, then

$$
\operatorname{sr}(A)=\max \left\{2, \operatorname{sr}\left(C\left(X_{\text {fin }}\right) \rtimes G\right)\right\} .
$$

Otherwise, $\operatorname{dim}\left(X_{H} / N_{G}(H)\right) \leq 1$ for all $H \in \mathcal{H} \backslash \mathcal{H}_{\text {fin }}$, and hence

$$
\operatorname{sr}(A)=\max \left\{1, \operatorname{sr}\left(C\left(X_{\text {fin }}\right) \rtimes G\right)\right\}=\max \left\{2, \operatorname{sr}\left(C\left(X_{\text {fin }}\right) \rtimes G\right)\right\} .
$$

Finally, if $\operatorname{dim}(X / G) \geq 1$, the stated formula for $\operatorname{RR}(A)$ follows in the same manner.

Recall that $X_{G}$ is the set of fixed points in $X$ under the action of $G$.

Corollary 2.5. Let $(G, X)$ be a transformation group, where $X$ is a second countable locally compact Hausdorff space and $G$ is a second countable compact group. Suppose that $X$ is locally of finite $G$-orbit type and that $\operatorname{dim}(X / G)<\infty$. Then

$$
\operatorname{sr}\left(C_{0}(X) \rtimes G\right) \leq 1+\left\lfloor\frac{1}{2} \operatorname{dim}(X / G)\right\rfloor .
$$

Suppose, in addition, that $G$ is connected. If $\operatorname{dim}(X / G) \leq 1$ then we have $\operatorname{sr}\left(C_{0}(X) \rtimes G\right)=1$, and if $\operatorname{dim}(X / G) \geq 2$ then

$$
\operatorname{sr}\left(C_{0}(X) \rtimes G\right)=\max \left\{2,1+\left\lfloor\frac{1}{2} \operatorname{dim}\left(X_{G}\right)\right\rfloor\right\},
$$

unless $X_{G}=\emptyset$, in which case $\operatorname{sr}\left(C_{0}(X) \rtimes G\right)=2$.

Proof. The first inequality follows from Theorem 2.3(b)(i), Theorem 2.4(i) and Lemma 1.5(ii). When $G$ is connected, $\mathcal{H}_{\text {fin }}$ is either empty or equal to $\{G\}$. If the action is trivial then Theorem 2.3(b)(i) yields $\operatorname{sr}\left(C_{0}(X) \rtimes G\right)=$ 
$1+\left\lfloor\frac{1}{2} \operatorname{dim} X\right\rfloor$ (which also follows from the fact that $C_{0}(X) \rtimes G \cong C_{0}(X) \otimes$ $C^{*}(G)$ ). If the action is non-trivial then Theorem 2.4(i) yields the required results.

Corollary 2.6. Let $G$ and $X$ be as in Corollary 2.5. Then

$$
\operatorname{RR}\left(C_{0}(X) \rtimes G\right) \leq \operatorname{dim}(X / G) .
$$

Suppose, in addition, that $G$ is connected. If $\operatorname{dim}(X / G)=0$ then we have $\operatorname{RR}\left(C_{0}(X) \rtimes G\right)=0$, and if $\operatorname{dim}(X / G) \geq 1$ then

$$
\operatorname{RR}\left(C_{0}(X) \rtimes G\right)=\max \left\{1, \operatorname{dim}\left(X_{G}\right)\right\},
$$

unless $X_{G}=\emptyset$, in which case $\operatorname{RR}\left(C_{0}(X) \rtimes G\right)=1$.

Proof. The proof is similar to that of Corollary 2.5, using Theorem 2.3(b)(ii) and Theorem 2.4(ii).

3. Motion group $C^{*}$-algebras. In [1] we have investigated the real and stable ranks for several classes of group $C^{*}$-algebras. However, these did not include $C^{*}$-algebras of motion groups. We are now able to treat some examples of motion groups as an application of Theorem 2.4.

For $n \geq 2$, let $\mathrm{SO}(n)$ act on $\mathbb{R}^{n}$ by rotation. Then $C_{0}\left(\mathbb{R}^{n}\right) \rtimes \mathrm{SO}(n)$ is isomorphic to the group $C^{*}$-algebra of the classical motion group $\mathbb{R}^{n} \rtimes \mathrm{SO}(n)$. More generally, let $K$ be any compact group and let $\alpha: k \mapsto \alpha_{k}$ be an injective homomorphism from $K$ into the automorphism group $\mathrm{GL}(n, \mathbb{R})$ of $\mathbb{R}^{n}$. Then, by replacing a given scalar product $\langle\cdot, \cdot\rangle$ on $\mathbb{R}^{n}$ by the scalar product $\langle\cdot, \cdot\rangle_{\alpha}$ defined by

$$
\langle v, w\rangle_{\alpha}=\int_{K}\left\langle\alpha_{k}(v), \alpha_{k}(w)\right\rangle d k, \quad v, w \in \mathbb{R}^{n},
$$

we can always assume that $K$ is a subgroup of $\mathrm{SO}(n)$.

In what follows, we consider $\mathrm{SO}(n-1)$ as embedded into $\mathrm{SO}(n)$ as the subgroup fixing the vector $v=(1,0, \ldots, 0) \in \mathbb{R}^{n}$.

TheOREM 3.1. Let $G$ be a connected closed subgroup of $\mathrm{SO}(n)$, where $n \geq 2$, and let $\left(\mathbb{R}^{n}\right)_{G}$ be the set of points in $\mathbb{R}^{n}$ which are fixed by $G$. Then

(i) $\operatorname{sr}\left(C_{0}\left(\mathbb{R}^{n}\right) \rtimes G\right)=1$ if and only if $\operatorname{GaSO}(n-1) a^{-1}=\mathrm{SO}(n)$ for some $a \in \mathrm{SO}(n)$, and

$$
\operatorname{sr}\left(C_{0}\left(\mathbb{R}^{n}\right) \rtimes G\right)=\max \left\{2,1+\left\lfloor\frac{1}{2} \operatorname{dim}\left(\left(\mathbb{R}^{n}\right)_{G}\right)\right\rfloor\right\}
$$

otherwise;

(ii) $\operatorname{RR}\left(C_{0}\left(\mathbb{R}^{n}\right) \rtimes G\right)=\max \left\{1, \operatorname{dim}\left(\left(\mathbb{R}^{n}\right)_{G}\right)\right\}$.

Proof. Note that $\operatorname{dim}\left(\mathbb{R}^{n} / G\right) \leq n$ by [22, Theorem 3.16]. Define $X=$ $\mathbb{R}^{n} \backslash\{0\}$ and let $q: X \rightarrow X / G$ denote the quotient map. Since $X / G$ is 
metrizable and $q$ is a closed surjection,

$$
\begin{aligned}
n & =\operatorname{dim} X \leq \operatorname{dim}(X / G)+\sup \left\{\operatorname{dim}\left(q^{-1}(q(x))\right): x \in X\right\} \\
& =\operatorname{dim}(X / G)+\sup \{\operatorname{dim}(G \cdot x): x \in X\} \\
& =\operatorname{dim}(X / G)+\sup \left\{\operatorname{dim}\left(G / S_{x}\right): x \in X\right\}
\end{aligned}
$$

by [23, Chapter 9, Proposition 2.6]. For each $x \in X, S_{x}=G \cap a \operatorname{SO}(n-1) a^{-1}$ for some $a \in \mathrm{SO}(n)$, and conversely every such subgroup of $G$ occurs as a stability group. Thus

$$
n \leq \operatorname{dim}(X / G)+\sup \left\{\operatorname{dim}\left(G /\left(G \cap a \operatorname{SO}(n-1) a^{-1}\right)\right): a \in \mathrm{SO}(n)\right\} .
$$

Suppose first that $\operatorname{dim}\left(G /\left(G \cap a \operatorname{SO}(n-1) a^{-1}\right)\right) \leq n-2$ for all $a \in \operatorname{SO}(n)$. Then $\operatorname{dim}\left(\mathbb{R}^{n} / G\right)=\operatorname{dim}(X / G) \geq 2$ and Corollaries 2.5 and 2.6 show that

$$
\operatorname{sr}\left(C_{0}\left(\mathbb{R}^{n}\right) \rtimes G\right)=\max \left\{2,1+\left\lfloor\frac{1}{2} \operatorname{dim}\left(\left(\mathbb{R}^{n}\right)_{G}\right)\right\rfloor\right\}
$$

and $\operatorname{RR}\left(C_{0}\left(\mathbb{R}^{n}\right) \rtimes G\right)=\max \left\{1, \operatorname{dim}\left(\left(\mathbb{R}^{n}\right)_{G}\right)\right\}$.

Now suppose that $\operatorname{dim}\left(G /\left(G \cap a \operatorname{SO}(n-1) a^{-1}\right)\right) \geq n-1$ for some $a \in$ $\mathrm{SO}(n)$. Then, since $C_{0}\left(\mathbb{R}^{n}\right) \rtimes a^{-1} G a$ and $C_{0}\left(\mathbb{R}^{n}\right) \rtimes G$ are isomorphic and

$$
a^{-1}\left[G /\left(G \cap a \operatorname{SO}(n-1) a^{-1}\right)\right] a=a^{-1} G a /\left(a^{-1} G a \cap \operatorname{SO}(n-1)\right),
$$

we can assume that $a$ is the identity of $\mathrm{SO}(n)$. Let $H=G \cap \mathrm{SO}(n-1)$ and $C=$ $G \mathrm{SO}(n-1) / \mathrm{SO}(n-1)$. Since $g H \mapsto g \mathrm{SO}(n-1)$ provides a homeomorphism between $G / H$ and the closed subspace $C$ of $\mathrm{SO}(n) / \mathrm{SO}(n-1)$, it follows that

$$
n-1 \leq \operatorname{dim}(G / H)=\operatorname{dim} C \leq \operatorname{dim}(\operatorname{SO}(n) / \operatorname{SO}(n-1))=n-1 .
$$

Hence $\operatorname{dim} C=n-1$. Since $\mathrm{SO}(n) / \mathrm{SO}(n-1)=S^{n-1}$ is locally homeomorphic to $\mathbb{R}^{n-1}$, it follows from [17, Theorem IV.5] and a compactness argument that $C$ has non-empty interior. Then $V=\left\{g \in G: g \mathrm{SO}(n-1) \in C^{\circ}\right\}$ is a non-empty open subset of $G$. This implies that

$$
C=\bigcup_{g \in G} g V \operatorname{SO}(n-1)=\bigcup_{g \in G} g C^{\circ}
$$

is open (and closed) in $S^{n-1}$, hence equal to $S^{n-1}$. It follows that $G \cdot \mathrm{SO}(n-1)$ $=\operatorname{SO}(n)$ and hence $G \cdot v=S^{n-1}$. Therefore $X / G=(0, \infty)$ and Corollaries 2.5 and 2.6 imply that $\operatorname{sr}\left(C_{0}\left(\mathbb{R}^{n}\right) \rtimes G\right)=1$ and $\operatorname{RR}\left(C_{0}\left(\mathbb{R}^{n}\right) \rtimes G\right)=$ $\max \left\{1, \operatorname{dim}\left(\left(\mathbb{R}^{n}\right)_{G}\right)\right\}$.

Regarding the proof above, note that $G \cdot \mathrm{SO}(n-1)=\mathrm{SO}(n)$ if and only if $G$ acts transitively on the sphere $S^{n-1}$. Actions with this property are described in [16]. We are grateful to M. C. Crabb for drawing our attention to this reference.

Corollary 3.2. For all $n \geq 2$, we have

$$
\operatorname{sr}\left(C^{*}\left(\mathbb{R}^{n} \rtimes \mathrm{SO}(n)\right)\right)=\operatorname{RR}\left(C^{*}\left(\mathbb{R}^{n} \rtimes \mathrm{SO}(n)\right)\right)=1 .
$$


In [29], Sudo has obtained the comparable result $\operatorname{sr}\left(C^{*}\left(\mathbb{R}^{n} \rtimes \operatorname{Spin}(n)\right)\right)$ $=1$, where $\operatorname{Spin}(n)$ is the simply connected covering group of $\mathrm{SO}(n)$.

\section{References}

[1] R. J. Archbold and E. Kaniuth, Stable rank and real rank for some classes of group $C^{*}$-algebras, Trans. Amer. Math. Soc. 357 (2005), 2165-2186.

[2] B. Blackadar, A stable cancellation theorem for simple $C^{*}$-algebras, Proc. London Math. Soc. (3) 47 (1983), 303-305.

[3] G. E. Bredon, Introduction to Compact Transformation Groups, Academic Press, London, 1972.

[4] L. G. Brown and G. K. Pedersen, $C^{*}$-algebras of real rank zero, J. Funct. Anal. 99 (1991), 131-149.

[5] K. R. Davidson, $C^{*}$-algebras by Example, Fields Inst. Monogr. 6, Amer. Math. Soc., Providence, RI, 1996.

[6] K. J. Dykema and P. de la Harpe, Some groups whose reduced $C^{*}$-algebras have stable rank one, J. Math. Pures Appl. 78 (1999), 591-608.

[7] S. Echterhoff, On transformation group $C^{*}$-algebras with continuous trace, Trans. Amer. Math. Soc. 343 (1994), 117-133.

[8] N. Elhage Hassan, Rang réel de certaines extensions, Proc. Amer. Math. Soc. 123 (1995), 3067-3073.

[9] J. M. G. Fell, A Hausdorff topology on the closed subsets of a locally compact nonHausdorff space, ibid. 13 (1962), 472-476.

[10] P. Green, The local structure of twisted covariance algebras, Acta Math. 140 (1978), 191-240.

[11] E. Hewitt and K. A. Ross, Abstract Harmonic Analysis I, Springer, Berlin, 1963.

[12] E. Kaniuth, Group $C^{*}$-algebras of real rank zero or one, Proc. Amer. Math. Soc. 119 (1993), 1347-1354.

[13] E. Kirchberg and S. Wassermann, Exact groups and continuous bundles of $C^{*}$ algebras, Math. Ann. 315 (1999), 169-203.

[14] H. Lin, An Introduction to the Classification of Amenable $C^{*}$-algebras, World Sci., Singapore, 2001.

[15] D. Montgomery, Orbits of highest dimension, in: Seminar on Transformation Groups, Ann. of Math. Stud. 46, Chapter IX, Princeton Univ. Press, Princeton, NJ, 1960, 117-131.

[16] D. Montgomery and H. Samelson, Transformation groups of spheres, Ann. of Math. (2) 44 (1943), 454-470.

[17] J. Nagata, Modern Dimension Theory, North-Holland, Amsterdam, 1965.

[18] M. Nagisa, Stable rank of some full group $C^{*}$-algebras of groups obtained by the free product, Internat. J. Math. 8 (1997), 375-382.

[19] M. Nagisa, H. Osaka and N. C. Phillips, Ranks of algebras of continuous $C^{*}$-valued functions, Canad. J. Math. 53 (2001), 979-1030.

[20] V. Nistor, Stable rank for a certain class of type I $C^{*}$-algebras, J. Operator Theory 17 (1987), 365-373.

[21] H. Osaka, Real rank of crossed products by connected compact groups, Bull. London Math. Soc. 27 (1995), 257-264.

[22] R. S. Palais, Slices and equivariant imbeddings, in: Seminar on Transformation Groups, Ann. of Math. Stud. 46, Chapter VIII, Princeton Univ. Press, Princeton, NJ, 1960, 101-115. 
[23] A. R. Pears, Dimension Theory of General Spaces, Cambridge Univ. Press, Cambridge, 1975.

[24] M. A. Rieffel, Dimension and stable rank in the $K$-theory of $C^{*}$-algebras, Proc. London Math. Soc. (3) 46 (1983), 301-333.

[25] - The cancellation theorem for projective modules over irrational rotation algebras, ibid. 47 (1983), 285-302.

[26] M. Rørdam, Classification of Nuclear $C^{*}$-algebras, Encyclopaedia Math. Sci. 126, Part I, Springer, Berlin, 2002.

[27] E. Schulz, The stable rank of crossed products of sectional $C^{*}$-algebras by compact Lie groups, Proc. Amer. Math. Soc. 112 (1991), 733-744.

[28] A. J.-L. Sheu, A cancellation theorem for projective modules over the group $C^{*}$ algebras of certain nilpotent Lie groups, Canad. J. Math. 39 (1987), 365-427.

[29] T. Sudo, Stable rank of the $C^{*}$-algebras of amenable Lie groups of type I, Math. Scand. 84 (1999), 231-242.

[30] T. Sudo and H. Takai, Stable rank of the $C^{*}$-algebras of solvable Lie groups of type I, J. Operator Theory 38 (1997), 67-86.

[31] D. P. Williams, Transformation group $C^{*}$-algebras with continuous trace, J. Funct. Anal. 41 (1981), 40-76.

[32] - , The topology on the primitive ideal space of transformation group $C^{*}$-algebras and C.C.R. transformation group $C^{*}$-algebras, Trans. Amer. Math. Soc. 266 (1981), $335-359$.

Department of Mathematical Sciences

Institut für Mathematik

University of Aberdeen

Universität Paderborn

Aberdeen AB24 3UE, Scotland, UK

E-mail: r.archbold@maths.abdn.ac.uk

D-33095 Paderborn, Germany

E-mail: kaniuth@math.uni-paderborn.de

Received March 16, 2005

Revised version February 2, 2006 